\title{
Restoring sagittal and frontal balance following posterior instrumented fusion
}

\author{
Ozgur Dede ${ }^{1}$, Muharrem Yazici ${ }^{2}$ \\ ${ }^{1}$ Department of Orthopaedic Surgery, UPMC Children's Hospital of Pittsburgh, Pittsburgh, PA, USA; ${ }^{2}$ Department of Orthopaedics and \\ Traumatology, Hacettepe University School of Medicine, Ankara, Turkey \\ Contributions: (I) Conception and design: All authors; (II) Administrative support: M Yazici; (III) Provision of study materials or patients: All authors; \\ (IV) Collection and assembly of data: All authors; (V) Data analysis and interpretation: All authors; (VI) Manuscript writing: All authors; (VII) Final \\ approval of manuscript: All authors. \\ Correspondence to: Muharrem Yazici, MD. Department of Orthopaedics and Traumatology, Hacettepe University School of Medicine, 06100 Sihhiye, \\ Ankara, Turkey. Email: mimyazici@gmail.com.
}

\begin{abstract}
Correction techniques for adolescent idiopathic scoliosis have evolved over the years as the understanding of the disease and the implant technology advanced. Utilization of the modern implant systems, along with the appropriate corrective maneuvers and selection of the appropriate levels of instrumentation, improves the outcomes after surgical treatment of adolescent idiopathic scoliosis. Coronal and sagittal balance are important predictors for successful posterior spine fusion. Many corrective methods have been developed to address the multiple facets of the spinal deformity and curve types. Treating surgeons should familiarize themselves with all of the techniques, their utility, and appropriate usage in different deformity settings. A literature review is provided with the best evidence available to achieve optimal deformity correction to help guide treating physicians.
\end{abstract}

Keywords: Sagittal balance; surgery; idiopathic scoliosis; coronal balance; spine fusion

Submitted Nov 10, 2019. Accepted for publication Dec 03, 2019.

doi: $10.21037 /$ atm.2019.12.68

View this article at: http://dx.doi.org/10.21037/atm.2019.12.68

\section{Introduction}

During the past decade, there have been very exciting developments in spinal deformity management. The surgical techniques for correction of scoliosis and the implants have changed over the years. However, posterior instrumented spine fusion is still the standard of care for the treatment of severe deformity in children with adolescent idiopathic scoliosis.

The evolution of surgical spinal deformity correction started with in situ non-instrumented fusion along with body cast correction. The instrumented fusion surgery, as we know today, started with Harrington rods and nonsegmental instrumentation, which provided correction with distraction and compression. Harrington rods revolutionized the deformity correction as it provided internal fixation that resulted in better correction and decreased the need for cast immobilization. The evolution continued with segmental instrumentation with sublaminar wires as popularized by Eduardo Luque. Segmental instrumentation put an end to cast or brace immobilization. Another revolution was with Yves Cotrel and Jean Dubousset with multiple hooks and lumbar pedicle screws, allowing for better correction of the deformity. The current implant systems are referred to as the 'fourth generation' that includes exclusive use of pedicle screws and may be supplemented with other implants as needed. Hooks, sublaminar wires, and bands are used variably to a lesser extent.

The surgical approach also went through different phases. Anterior approach initially was mostly used for stiff curves to provide additional mobilization of the spine. Towards the end of the $20^{\text {th }}$ century, anterior approach was utilized in isolation along with instrumentation for selected 
curves. Eventually, with the commonplace use of pedicle screws in the thoracic and lumbar spine, anterior approach is rarely used for very stiff curves for added mobilization with discectomies. Fusion, along with a safe amount of deformity correction, is the ultimate goal and involves utilizing local and/or allograft bone for the majority of cases.

This manuscript will discuss the current posterior surgical techniques for sagittal and coronal deformity correction in children with adolescent idiopathic scoliosis. The decision-making points that are described in this manuscript are fashioned for adolescent idiopathic scoliosis and should not be generalized to other types of scoliosis.

\section{Restoration of coronal and sagittal balance}

Restoration of the coronal and sagittal balance requires selection of appropriate levels for instrumentation, providing sufficient correction, and utilizing one or more corrective maneuvers and selection of the appropriate implant materials.

The surgeon must decide on which implants and corrective techniques to employ depending on the size, location, and the stiffness of the curve. The surgeon's preference, and experience often do and should play a role in these decisions as well. If there is sagittal deformity such as kyphosis or lordosis, this will also affect the choice of fusion levels, corrective techniques, and potentially the implant choice.

\section{Level selection}

Level selection is probably the most important step once the surgical treatment decision is made. Appropriate level selection will help provide optimal post-operative spinal balance as well as hopefully satisfactory residual spinal mobility.

A brief discussion of spinal deformity classification is worthwhile. The most commonly cited two classification systems for adolescent idiopathic scoliosis are the King and Moe classification and the Lenke classification system $(1,2)$. Lenke system provides a more in-depth description of a spinal deformity in both coronal and sagittal planes and therefore is the classification of choice today. However, King and Moe's system deserves some mention as it provides a good understanding of the common deformities and describes a curve that is not well characterized by the Lenke system.
We utilize the Lenke classification as a guide to decide on which curves to instrument. All structural curves usually have to be included in the fusion. The main exceptions include type $1 \mathrm{C}$ and type 3 curves. In both instances, the decision to only fuse the main thoracic curves in order to leave more spinal mobility (selective thoracic fusion) versus addressing both curves for the sake of better correction and balance will have to be made. Generally, in type 3 curves, the lumbar curve should be included in the posterior fusion. Successful selective thoracic fusion requires the thoracic curve to be larger than the lumbar curve $(>1.2)$, apical rotation of the thoracic curve to be more severe, and the apical vertebral translation of the thoracic curve to be more than the lumbar curve (>1.2).

Additionally, if there is already a preoperative trunk shift to the left or a very large lumbar rotation which might be either radiological or clinical lumbar prominence or curve $>60$ degrees, apical vertebral translation over $4 \mathrm{~cm}$ or $>$ grade 2.5 rotation (Nash and Moe), selective thoracic fusion may not be a good option. The presence of sagittal plane deformity and skeletal immaturity may also preclude a selective thoracic fusion. If these criteria are followed, usually, the lumbar spine may be spared with a successful coronal balance in the long term (3-5). However, the readers are forewarned that this is not a fail-proof method. Even following these criteria strictly, there will be some lumbar curves that progress or decompensate. Selective thoracic fusion will likely be a topic of debate for many years to come.

\section{Curves types and level selection}

\section{Lenke type 1, King and Moe type 3 and some King and Moe type 2}

Main thoracic curves are the most typical idiopathic scoliosis curves. The common conception is to include both end vertebrae in the fusion. Central sacral line, stable vertebra, or stable vertebra-to-be on supine traction radiographs may be utilized to decide on the distal extent of the instrumentation. The most cranial vertebra touched by the central sacral vertical line is the most common recommendation for the lower instrumented vertebra. Some evidence suggests that supine traction under general anesthesia may provide more information and help save about 1 level (6). For this method, the distal-most vertebra on the curve that centralized on supine traction radiograph under anesthesia is utilized. For the proximal extent, 
common recommendations are that if the left shoulder is lower than the right shoulder, often the upper instrumented level is chosen as T4. If the shoulders are level then UIV is $\mathrm{T} 3$ and if the left shoulder is higher than the right shoulder $\mathrm{T} 2$ is typically chosen as the upper instrumented vertebra. For Lenke type $1 \mathrm{C}$ curves, the decision to include or spare the lumbar curve can be made using the criteria listed previously for selective thoracic fusion.

\section{Lenke type 2, King and Moe type 5}

These are double thoracic curves, and typically, the fusion extends to T2 on the proximal aspect. The distal level selection is the same as the type 1 curves.

\section{Lenke type 3, some King and Moe type 2}

For these double major curves, both curves should be included in the fusion. In rare cases, selective thoracic fusion may be contemplated; however, the usual level selection includes both curves. Typical fusion levels are T4 to $\mathrm{L} 3$ or L4.

\section{Lenke type 4}

This is a triple major curve where all three curves will need to be addressed. Typical levels for posterior spinal fusion would include T2 to L3 or L4.

\section{Lenke type 5}

This is the thoracolumbar or lumbar curve. Proximal level selection should be based on the end vertebra as well as the sagittal alignment. Distally, L4, and rarely L5 may need to be considered.

\section{Lenke type 6, King and Moe type 1}

This curve pattern has a double curve pattern with the lumbar curve being larger than the thoracic curve; however, both structural. Both curves often need to be addressed for optimal correction.

\section{King type 4}

This curve pattern was not initially described in the Lenke classification system; however, a modification was proposed by calling this curve pattern Lenke $1 \mathrm{AR}$ (7). This is a long thoracic curve with a lower apex and should be fused down more distally, typically to L3 or L4, to prevent postoperative trunk shift.

\section{Sagittal plane evaluation}

Sagittal evaluation should always be part of preoperative evaluation. The reader is urged to always check the lateral radiographs even in the most typical adolescent idiopathic scoliosis case. Level selection should always be decided upon only after evaluation of the sagittal alignment of the spine. As an example, for a typical main thoracic curve, if the child has kyphosis at the thoracolumbar junction, the fusion level will have to be moved distally to include the kyphotic segment. Spondylolisthesis is not a very uncommon issue and may affect the level selection and even the surgical decision. The lateral radiograph should be closely evaluated in order not to miss a pars defect or a lowgrade spondylolisthesis.

Sagittal stable vertebra concept has been scrutinized recently and has been noted to have some importance on level selection to avoid junctional kyphosis (8).

\section{Implant and metal choice}

There are a variety of implants that can be utilized to anchor the rods to the vertebrae. Hooks, wires, sublaminar wires/ bands, and pedicle screws are the currently used instruments with pedicle screws being the most common implant of choice. Hooks can be used at the top of the instrumentation in an attempt to decrease the likelihood of proximal junctional kyphosis; however, it is not known if this is truly beneficial (9). Otherwise, hooks, wires, and bands are often used as alternative or salvage fixation implants.

The material choice may affect the amount of correction. Three major rod options are stainless steel, titanium (Ti) alloy, and cobalt-chromium (Co-Cr) alloy. Ti has the most give and is the one with the lowest modulus of elasticity, therefore tends to bend into the curve. This may be overcome to a certain extent by increasing the diameter. In healthy bone, if more correction is desired using Co-Cr or stainless steel may be considered. There is some evidence pointing towards less infection incidence with the use of $\mathrm{Ti}$ alloys in posterior spine fusion.

\section{Correction techniques}

Several correction techniques are available. Such techniques 
can be used independently or jointly, depending on curve pattern and severity.

\section{Rod de-rotation}

This is one of the classical and well-accepted ways to correct spinal deformity. Rod de-rotation was not possible before the time of multiple hooks and screw fixation. Harrington instrumentation provided correction with compression and distraction forces. Sublaminar wiring, as popularized by Eduardo Luque, predominantly utilized the translation forces as the wires were tightened. Only after the Cotrel-Dubousset multiple hook/screw constructs were introduced, rod de-rotation became one of the main corrective maneuvers for spinal deformity, and it is still utilized frequently (10).

\section{Vertebral de-rotation, en-bloc and direct}

The premise of vertebral derotation is to correct the axial deformity or torsion of the spine (11). The technique can be utilized either separately on each vertebral level (direct) or the whole apical segment (en-bloc) of the curve. Once the coronal correction is completed, and the concave rod is in place (or both rods), the proximal or distal levels are locked with set screws to the rod. This is important as otherwise, the whole spine will de-rotate. Following this step, derotating instruments are placed on the apical screws, which is the area of maximal axial deformity. With the proximal or distal segments are locked and the intervening set screws loose, the apical vertebrae are derotated in order to decrease the thoracic or lumbar prominence. This can be achieved either level by level or could be done as the whole apical segment. The overall goal is to decrease the torsional deformity that causes the rib prominence or lumbar muscle prominence.

\section{Differential rod contouring}

This technique may supplement any other type of corrective maneuver as it is simply contouring of the rods. Once the rods are cut to the proper length, the concave and convex rods are contoured slightly (sometimes not so slightly) differently. The concave sided rod for a typical thoracic curve would be contoured with exaggerated kyphosis, and the convex rod will be contoured with a less kyphosis (or as hypokyphotic) —sometimes almost straight. The idea here is to help pull the concavity up to a more normalized sagittal contour as well as to 'push' the increased rotation of the convexity 'down' (anteriorly) to help with the 'de-rotation' of the spinal deformity.

\section{Cantilever maneuver}

The cantilever maneuver is mainly used for neuromuscular curves that extend to the pelvis. This is a very useful maneuver to correct the pelvic obliquity. The usefulness in adolescent idiopathic scoliosis surgery is very limited. However, with double thoracic curves, this technique may be used to correct the upper thoracic curve with bilateral rods and then cantilever these rods to the distal pedicle screws in order to correct the more distal curve.

\section{Coronal and sagittal translation}

This maneuver consists of pulling the vertebrae to the rod. The appropriately contoured rods are placed in their final position, and the vertebrae are reduced to the rods gradually. This technique was the main correction technique in the era of sublaminar wires as well as the unit rod for neuromuscular scoliosis. Currently, pedicle screws are utilized and pulled to the rods using reduction devices or reduction tabs. For this technique to be successful, the rods should be from a stiffer grade, such as stainless steel or Co-Cr. Ti often bends into the lordosis of the deformity on the concave side of the thoracic spine. For the application of the technique, the rod is placed into the very proximal and distal implants, and one side is fixed, and the other side is loosely closed with set screws. Then the in-between segments where the deformity is largest are pulled to the rod using reduction tools or tabs of the screws. The reduction has to be gradual as screw pull-out is a likely complication.

\section{Compression and distraction}

Once were utilized as the main part of the corrective and fixation technique, these maneuvers have a limited role in the age of pedicle screw instrumentation. However, compression and distraction may still be used at the distal and proximal ends of the instrumentation to help level the end vertebrae. Specifically, for the distal end of the instrumentation, compression should be applied to the corrective side first, then distraction on the contralateral side for leveling of that segment. Large amounts of compression may lead to lordosis or hypokyphosis. On 
the other hand, large amounts of distraction may lead to increased kyphosis or loss of lordosis. In the pedicle screw era, since the use of compression and distraction is only limited to fine-tuning, the kyphosing or lordosing effects are likely to be negligible.

\section{In-situ rod contouring on the coronal and sagittal plane}

This method can be used to fine-tune the correction that is already achieved after the placement of the rods. The metal choice is important. Ti alloys are not a very good option if this correction technique is to be utilized. There are two reasons; one being $\mathrm{Ti}$ has the disadvantage of notch sensitivity. Too many bends may render the Ti to fail prematurely. The other reason is that Ti needs to be bent quite a bit to change the shape, as it tends to spring back to a certain extent. Stainless steel is a better implant choice for in-situ rod contouring, although it is a less used material when compared to two decades ago.

\section{Temporary rods}

This is a not very commonly used technique as satisfactory correction can be achieved in most instances without exchanging or using temporary rods. One specific deformity that lends itself for this technique is double thoracic curves (Lenke 2, King 5), where there is a proximal left-sided curve and a main right thoracic curve. Applying a pre-bent single rod and de-rotation would be counterproductive for a double thoracic curve, as this would cause unwanted lordosis of the proximal thoracic curve. Therefore, a temporary initial rod can be cut to the length of the proximal thoracic curve, placed on the concave side of the proximal thoracic curve, and de-rotated. This will provide a tentative correction for the proximal thoracic curve. Then, on the concave side of the main thoracic curve (left side of the patient), a rod is fashioned similar to the technique utilized for a single thoracic curve. This second rod is placed utilizing the typical corrective maneuvers, either rod de-rotation or translation techniques, pulling the spine to the rod.

\section{Clinical outcomes}

The clinical outcomes after posterior spine fusion are very good as long as the common guidelines for deformity correction and level choice are applied. The difficulty arises in cases with severe preoperative shoulder imbalance as well as double curves where a selective thoracic fusion is intended. Uninstrumented curve worsening with continued growth or trunk decompensation are problems that are not completely resolved.

\section{Conclusions}

Surgical treatment of adolescent idiopathic scoliosis is a continuously evolving field. For optimal patient outcomes, the surgeons should be able to utilize all available correction techniques and implants and tailor the treatment according to the specific deformity pattern. Fusionless or limited fusion techniques are being researched and are potentially expected to become the standard treatment in the near future.

\section{Acknowledgments}

None.

\section{Footnote}

Conflicts of Interest: The authors have no conflicts of interest to declare.

Ethical Statement: The authors are accountable for all aspects of the work in ensuring that questions related to the accuracy or integrity of any part of the work are appropriately investigated and resolved.

\section{References}

1. King HA, Moe JH, Bradford DS, et al. The selection of fusion levels in thoracic idiopathic scoliosis. J Bone Joint Surg Am 1983;65:1302-13.

2. Lenke LG, Betz RR, Harms J, et al. Adolescent idiopathic scoliosis: a new classification to determine extent of spinal arthrodesis. J Bone Joint Surg Am 2001;83:1169-81.

3. Lenke LG, Bridwell KH, Baldus C, et al. Preventing decompensation in King type II curves treated with CotrelDubousset instrumentation. Strict guidelines for selective thoracic fusion. Spine (Phila Pa 1976) 1992;17:S274-81.

4. Louer C Jr, Yaszay B, Cross M, et al. Ten-year outcomes of selective fusions for adolescent idiopathic scoliosis. J Bone Joint Surg Am 2019;101:761-70.

5. Newton PO, Faro FD, Lenke LG, et al. Factors involved in the decision to perform a selective versus nonselective fusion of Lenke 1B and 1C (King-Moe II) curves in 


\section{Page 6 of 6}

adolescent idiopathic scoliosis. Spine (Phila Pa 1976) 2003;28:S217-23.

6. Hamzaoglu A, Ozturk C, Enercan M, et al. Traction X-ray under general anesthesia helps to save motion segment in treatment of Lenke type $3 \mathrm{C}$ and $6 \mathrm{C}$ curves. Spine J 2013;13:845-52.

7. Miyanji F, Pawelek JB, Van Valin SE, et al. Is the lumbar modifier useful in surgical decision making?: defining two distinct Lenke 1A curve patterns. Spine (Phila Pa 1976) 2008;33:2545-51.

8. Yang J, Andras LM, Broom AM, et al. Preventing distal junctional kyphosis by applying the stable sagittal vertebra concept to selective thoracic fusion in adolescent idiopathic

\section{Dede and Yazici. Restoring balance after posterior spine fusion}

scoliosis. Spine Deform 2018;6:38-42.

9. Pahys JM, Vivas AC, Samdani AF, et al. Assessment of proximal junctional kyphosis and shoulder balance with proximal screws versus hooks in posterior spinal fusion for adolescent idiopathic scoliosis. Spine (Phila Pa 1976) 2018;43:E1322-8.

10. Dubousset J, Cotrel Y. Application technique of CotrelDubousset instrumentation for scoliosis deformities. Clin Orthop Relat Res 1991;(264):103-10.

11. Lee SM, Suk SI, Chung ER. Direct vertebral rotation: a new technique of three-dimensional deformity correction with segmental pedicle screw fixation in adolescent idiopathic scoliosis. Spine (Phila Pa 1976) 2004;29:343-9.
Cite this article as: Dede O, Yazici M. Restoring sagittal and frontal balance following posterior instrumented fusion. Ann Transl Med 2020;8(2):30. doi: 10.21037/atm.2019.12.68 